$\mathrm{UG}-10 / 94$

FIAN/TD/17/94

hep-th/9411093

November 1994

\title{
The Calogero Model and the Virasoro Symmetry
}

\author{
E. Bergshoeff \\ Institute for Theoretical Physics \\ Nijenborgh 4, 9747 AG Groningen \\ The Netherlands \\ and \\ M. Vasiliev" \\ I.E. Tamm Theoretical Department, P.N. Lebedev Physical Institute \\ 117924, Leninsky Prospect 53, Moscow \\ Russia
}

\begin{abstract}
We construct new realizations of the Virasoro algebra inspired by the Calogero model. The Virasoro algebra we find acts as a kind of spectrumgenerating algebra of the Calogero model. We furthermore present the superextension of these results and introduce a class of higher-spin extensions of the Virasoro algebra which are of the $W_{\infty}$ - type.
\end{abstract}

\footnotetext{
${ }^{1}$ Supported in part by the Russian Fund of Fundamental Research, grant N67123016.
} 


\section{Introduction}

Over the years there has been an increasing interest in the possible relationships between integrable systems and conformal field theory. A well-known and well studied example is provided by the Liouville model and, more generally, the Toda models which play a crucial role in the study of non-critical string theories.

Another example of an integrable system is the Calogero model which has been studied intensely since its construction in 1969 [1, 2]. Recently, further progress has been obtained in understanding the $N$-body Calogero model with harmonic interaction [3, 4]. In [5, 6, 7] it was argued that the Calogero model describes one-dimensional reductions of anyonic systems 8, 9], e.g. anyons at the lowest Landau level in a strong magnetic field. In its turn, anyon physics plays an important role in understanding the (fractional) quantum Hall effect (QHE) [10] that assumes interesting links between the latter and the Calogero model.

The recent progress [3, 4] in the understanding of the (rational) Calogero model was based on an extension of the Heisenberg algebra, the so-called $S H_{N}(\nu)$ algebra [11. For $N=1$ the algebra reduces to the ordinary Heisenberg algebra which underlies the higher-spin algebras in higher dimensions [12 as well as the standard realization of the Virasoro algebra as vector fields and of the $W_{1+\infty}$ algebra [13] as differential operators on the circle.

The question we address in this paper is whether the general $S H_{N}(\nu)$ algebra can lead to new realizations of the (super) Virasoro algebra. We show that this is indeed the case and, as a result, one discovers a new interesting class of $W_{\infty}$ - type algebras containing higher-spin currents in addition to the Virasoro generators. We expect that our results will lead to a better insight into possible relations between the Calogero model and conformal field theory. Our results may also have applications to the quantum Hall effect. Actually, it was observed recently [14] that the relevant approach to the QHE is based on the representation theory of the $W_{1+\infty}$ algebra developed in [15]. Since, on the other hand, the algebras considered in the present paper are responsible for the $N$ - body excitations in the Calogero model and can be regarded as some (extensions $\times$ deformations) of the ordinary $W_{1+\infty}$ algebra

one can speculate that they might be relevant for the analysis of the manybody excitations in the QHE. 


\section{The $S H_{N}(\nu)$ realization of the Virasoro Al- gebra}

Our starting point is the $S_{N}$-extended Heisenberg algebra $S H_{N}(\nu)$ [3, 4, 11] which can be regarded as the algebra formed by the generating elements $a_{i}, a_{i}^{\dagger}$ and $K_{i j} \quad(i, j=1, \ldots, N)$ obeying the following relations?:

$$
\begin{aligned}
{\left[a_{i}^{(\dagger)}, a_{j}^{(\dagger)}\right] } & =0, \quad\left[a_{i}, a_{j}^{\dagger}\right]=A_{i j} \equiv \delta_{i j}\left(1+\nu \sum_{l=1}^{N} K_{i l}\right)-\nu K_{i j}, \\
K_{i j} K_{j l} & =K_{j l} K_{i l}=K_{i l} K_{i j}, \quad \text { for all } i \neq j, i \neq l, j \neq l, \\
\left(K_{i j}\right)^{2} & =I, \quad K_{i j}=K_{j i}, \\
K_{i j} K_{m n} & =K_{m n} K_{i j}, \quad \text { if all indices } i, j, m, n \text { different }, \\
K_{i j} a_{j}^{(\dagger)} & =a_{i}^{(\dagger)} K_{i j} .
\end{aligned}
$$

Here $\nu$ is a constant related to the Calogero coupling constant, while $K_{i j}$ are the elementary permutation operators of the $S_{N}$ exchange algebra. We use the standard convention that square brackets denote commutators and curly brackets anticommutators.

To make contact between the $S H_{N}(\nu)$ algebra and the Calogero model $]^{3}$ one has to use the following "Calogero realization" of $S H_{N}(\nu)$ :

$$
a_{i}=\frac{1}{\sqrt{2}}\left(x_{i}+D_{i}\right), \quad a_{i}^{\dagger}=\frac{1}{\sqrt{2}}\left(x_{i}-D_{i}\right),
$$

with

$$
D_{i}=\frac{\partial}{\partial x_{i}}+\nu \sum_{j \neq i}\left(x_{i}-x_{j}\right)^{-1}\left(1-K_{i j}\right) .
$$

The real Calogero coordinates $x_{i}$ and the so-called Dunkl derivatives $D_{i}$ [17] can be shown, by a direct calculation, to satisfy the commutation relations [3, 舟:

$$
\left[x_{i}, x_{j}\right]=\left[D_{i}, D_{j}\right]=0, \quad\left[D_{i}, x_{j}\right]=A_{i j} .
$$

The crucial observation that establishes the relation with the Calogero model is that the Calogero Hamiltonian $H_{C a l}$ is related to the operator

$$
H=\frac{1}{2} \sum_{i=1}^{N}\left\{a_{i}, a_{i}^{\dagger}\right\} .
$$

\footnotetext{
${ }^{2}$ In this paper repeated indices do not imply summation.

${ }^{3}$ For a review of the Calogero model as a classical and quantum integrable model, see [16.
} 
This operator, like the ordinary harmonic oscillator Hamiltonian, obeys the standard relations

$$
\left[H, a_{i}^{\dagger}\right]=a_{i}^{\dagger}, \quad\left[H, a_{i}\right]=-a_{i} .
$$

The operator $H$ is called the universal Calogero Hamiltonian because it also can be used to describe spinning Calogero models [18, 19]. The precise relationship with the original Calogero Hamiltonian $H_{C a l}$ involves a simple similarity transformation followed by a restriction to the subspace of totally symmetric wave functions. This construction allows one to construct all eigen wave functions of the model as the Fock vectors

$$
\left(a_{1}^{\dagger}\right)^{n_{1}} \ldots\left(a_{N}^{\dagger}\right)^{n_{N}}|0\rangle .
$$

For more details, we refer to [4].

Our goal in this section is to investigate whether the $S H_{N}(\nu)$ algebra defined in eqs. (1)-(5) can lead to new realizations of the Virasoro algebra. Our starting point is the following Ansatz for the Virasoro generators :

$$
L_{-n}=\sum_{i=1}^{N}\left(\alpha\left(a_{i}^{\dagger}\right)^{n+1} a_{i}+\beta a_{i}\left(a_{i}^{\dagger}\right)^{n+1}+\left(\lambda-\frac{1}{2}\right)(n+1)\left(a_{i}^{\dagger}\right)^{n}\right),
$$

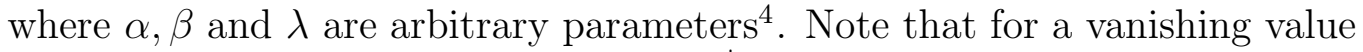
of the parameter $\nu$ we have that $\left[a_{i}, a_{j}^{\dagger}\right]=\delta_{i j}$ and the above generators correspond to a direct sum of the $N$ standard vector field generators of the Virasoro algebra. Remarkably, it turns out that the Ansatz (12) also works for non-vanishing values of $\nu$. In order to prove this it is convenient to sum over all the modes and to rewrite the Ansatz for the Virasoro generators in terms of a parameter function $\xi\left(a_{i}^{\dagger}\right)$ as

$$
L_{\xi}=\sum_{i=1}^{N}\left(\alpha \xi\left(a_{i}^{\dagger}\right) a_{i}+\beta a_{i} \xi\left(a_{i}^{\dagger}\right)+\left(\lambda-\frac{1}{2}\right) \frac{\partial}{\partial a_{i}^{\dagger}} \xi\left(a_{i}^{\dagger}\right)\right) .
$$

The proof that the Ansatz (13) satisfies the Virasoro algebra commutation relations requires the following three identities

$$
\begin{aligned}
\sum_{i=1}^{N} \xi_{1}\left(a_{i}^{\dagger}\right)\left[a_{i}, \xi_{2}\left(a_{j}^{\dagger}\right)\right]-(1 \leftrightarrow 2) & =\xi_{1}\left(a_{j}^{\dagger}\right) \frac{\partial}{\partial a_{j}^{\dagger}} \xi_{2}\left(a_{j}^{\dagger}\right)-(1 \leftrightarrow 2), \\
\sum_{i=1}^{N}\left[a_{i}, \xi_{1}\left(a_{j}^{\dagger}\right)\right] \xi_{2}\left(a_{i}^{\dagger}\right)-(1 \leftrightarrow 2) & =\frac{\partial}{\partial a_{j}^{\dagger}} \xi_{1}\left(a_{j}^{\dagger}\right) \xi_{2}\left(a_{j}^{\dagger}\right)-(1 \leftrightarrow 2), \\
\sum_{i, j=1}^{N}\left[\left[a_{i}, \xi_{1}\left(a_{i}^{\dagger}\right)\right],\left[a_{j}, \xi_{2}\left(a_{j}^{\dagger}\right)\right]\right] & =0,
\end{aligned}
$$

\footnotetext{
${ }^{4}$ For $N=1$ and $\alpha=\beta=1 / 2$ the parameter $\lambda$ coincides with the $\lambda$ of 20.
} 
where $\xi_{1}$ and $\xi_{2}$ are arbitrary Laurent series. The proof of the identities (14), (15) and (16) is given in appendix A. It is based on the following useful formula:

$$
\left[a_{i}, f\left(a^{\dagger}\right)\right]=\frac{\partial}{\partial a_{i}^{\dagger}} f\left(a^{\dagger}\right)-\nu \sum_{l=1}^{N}\left(a_{i}^{\dagger}-a_{l}^{\dagger}\right)^{-1}\left[K_{i l}, f\left(a^{\dagger}\right)\right] .
$$

This formula is a direct consequence of the basic commutation relations (1) and can be easily proven by expanding $f\left(a^{\dagger}\right)$ in a Laurent series in $a_{i}^{\dagger}$. Alternatively, one can observe that the right-hand-side of (17) $(i)$ respects the Leibniz rule, $(i i)$ vanishes when $f=$ const and (iii) reduces to the basic commutation relation (1) for $f=a_{i}^{\dagger}$. Combined altogether the properties (i)-(iii) prove that the formula (17) is valid for an arbitrary polynomial in $a_{i}^{\dagger}$ and $\left(a_{i}^{\dagger}\right)^{-1}$.

Note that the right-hand-side of (17) remains regular for regular functions $f\left(a^{\dagger}\right)$, i.e. the poles in $\left(a_{i}^{\dagger}-a_{l}^{\dagger}\right)$ cancel due to the commutator with $K_{i l}$. It is worth mentioning that the formula (17) can be used for a simple derivation of the Dunkl derivative and its further generalizations given in [11] as the action of the $a_{i}$ - type operators in the Fock modules with the vacuum states satisfying the conditions $a_{i}|0\rangle=0, K_{i j}|0\rangle=T_{i j}|0\rangle$ where the matrices $T_{i j}$ realize some representation $t$ of the symmetric group $S_{N}$ which acts on the vacuum vector(s) $|0\rangle$. The Dunkl derivatives then correspond to the trivial representation of $S_{N}, T_{i j}=1$, while the derivatives introduced in [11] correspond to a general representation $t$.

We now proceed with the proof that the Ansatz (13) satisfies the Virasoro algebra commutation relations. For simplicity, we first consider the special case with $\lambda=1 / 2$. Using the standard Leibniz rule we find that

$$
\begin{aligned}
{\left[L_{\xi_{1}}, L_{\xi_{2}}\right]=} & \sum_{i, j=1}^{N}\left(\alpha^{2} \xi_{1}\left(a_{i}^{\dagger}\right)\left[a_{i}, \xi_{2}\left(a_{j}^{\dagger}\right)\right] a_{j}-\beta^{2} a_{j}\left[a_{i}, \xi_{1}\left(a_{j}^{\dagger}\right)\right] \xi_{2}\left(a_{i}^{\dagger}\right)\right. \\
& \left.+\alpha \beta\left[\xi_{1}\left(a_{i}^{\dagger}\right) a_{i}, a_{j} \xi_{2}\left(a_{j}^{\dagger}\right)\right]\right)-(1 \leftrightarrow 2) .
\end{aligned}
$$

The third term at the right-hand-side can be rewritten as

$$
\begin{aligned}
\alpha \beta \sum_{i, j=1}^{N}( & \frac{1}{2}\left[\xi_{1}\left(a_{i}^{\dagger}\right) a_{i}, \xi_{2}\left(a_{j}^{\dagger}\right) a_{j}\right]+\frac{1}{2}\left[a_{j} \xi_{1}\left(a_{j}^{\dagger}\right), a_{i} \xi_{2}\left(a_{i}^{\dagger}\right)\right] \\
& \left.-\left[\left[a_{i}, \xi_{1}\left(a_{i}^{\dagger}\right)\right],\left[a_{j}, \xi_{2}\left(a_{j}^{\dagger}\right)\right]\right]\right)-(1 \leftrightarrow 2)
\end{aligned}
$$

The last term in this expression vanishes due to the identity (16) while the first two terms are identical to the contribution from the $\alpha^{2}$ and $\beta^{2}$ terms. Application of the identities (14), (15) to the remaining terms gives the result 


$$
\left[L_{\xi_{1}}, L_{\xi_{2}}\right]=(\alpha+\beta) \sum_{i=1}^{N}\left(\alpha \xi_{1,2}\left(a_{i}^{\dagger}\right) a_{i}+\beta a_{i} \xi_{1,2}\left(a_{i}^{\dagger}\right)\right)
$$

with the parameter $\xi_{1,2}\left(a_{i}^{\dagger}\right)$ given by

$$
\xi_{1,2}\left(a_{i}^{\dagger}\right)=\xi_{1}\left(a_{i}^{\dagger}\right) \frac{\partial}{\partial a_{i}^{\dagger}} \xi_{2}\left(a_{i}^{\dagger}\right)-\xi_{2}\left(a_{i}^{\dagger}\right) \frac{\partial}{\partial a_{i}^{\dagger}} \xi_{1}\left(a_{i}^{\dagger}\right) .
$$

We conclude that the Ansatz (12) leads to the Virasoro algebra provided that

$$
\alpha+\beta=1 .
$$

This result can easily be extended to other values of $\lambda$ with $\lambda \neq 1 / 2$ by the use of only the identities (14) and (15).

Thus it is shown that there exists a two-parametric class of realizations of the Virasoro algebra constructed from the Calogero oscillators which are the generating elements of $S H_{N}(\nu)$. We now make some comments to the above result:

(1) The proof that the generators (13) form the Virasoro algebra is based entirely on the commutation relations (1)-(5) and is independent of any particular realization of this algebra. By their construction the generators are invariant under the action of the symmetric group

$$
K_{i j} L_{\xi}=L_{\xi} K_{i j} .
$$

Among other things this means that the Virasoro algebra closes and the above $S_{N}$-invariance property remains valid if the parameters $\alpha$ and $\lambda$ are not just pure numbers but depend on any combination of group algebra elements of $S_{N}$ which are $S_{N}$ invariant themselves, i.e.

$$
\begin{gathered}
\alpha=\alpha\left(T_{n}\right) ; \quad \lambda=\lambda\left(T_{n}\right), \\
T_{n}=\sum_{i_{1} \neq i_{2} \neq \ldots \neq i_{n}} K_{i_{1} i_{2}} K_{i_{2} i_{3}} \ldots K_{i_{n} i_{1}} .
\end{gathered}
$$

In the discussion below we will only need the case that $\alpha$ is constant and $\lambda$ is at most linear in $K_{i j}$ :

$$
\alpha=\alpha_{0}, \quad \lambda=\lambda_{0}+\lambda_{1} \sum_{i \neq j} K_{i j},
$$

\footnotetext{
${ }^{5}$ Particular examples of this class has been known to other authors: S. Isakov and J. Leinaas have studied the case with $\alpha=\beta=1 / 2$, while the fact that the Virasoro algebra closes for the cases with $\alpha=0$ or $\beta=0$ was pointed out to us by A. Polychronakos (private communications).
} 
where $\alpha_{0}, \lambda_{0}$ and $\lambda_{1}$ are some constants. This freedom allows us to define the $L_{0}$ generator of the Virasoro algebra in such a way that it satisfies the properties of a $Z$-grading operator for the whole enveloping algebra of $S H_{N}(\nu)$, i.e. it satisfies the same identities as the universal Calogero Hamiltonian (see $(10))$.

The generators $\left\{L_{1}, L_{0}, L_{-1}\right\}$ which are given by

$$
\begin{aligned}
L_{1} & =\sum_{i=1}^{N} a_{i} \\
L_{0} & =\sum_{i=1}^{N}\left(\alpha a_{i}^{\dagger} a_{i}+(1-\alpha) a_{i} a_{i}^{\dagger}\right)+\left(\lambda-\frac{1}{2}\right) N, \\
L_{-1} & =\sum_{i=1}^{N}\left(\alpha\left(a_{i}^{\dagger}\right)^{2} a_{i}+(1-\alpha) a_{i}\left(a_{i}^{\dagger}\right)^{2}\right)+2\left(\lambda-\frac{1}{2}\right) \sum_{i=1}^{N} a_{i}^{\dagger},
\end{aligned}
$$

form a $\operatorname{sl}(2)$ subalgebra of the Virasoro algebra. $L_{1}$ coincides with the annihilation operator of the center of mass degree of freedom while $L_{0}$ is nothing else than the universal Calogero Hamiltonian (9) modulo terms which become a constant when acting on the subspace of symmetric wavefunctions, i.e.:

$$
L_{0}-H=(\lambda-\alpha) N-\nu\left(\alpha-\frac{1}{2}\right) \sum_{i \neq j} K_{i j} .
$$

One can fix the free parameteres $\alpha$ and $\lambda$ to be

$$
\alpha=\alpha_{0} \quad \lambda=\lambda_{0}+\nu N^{-1}\left(\alpha-\frac{1}{2}\right) \sum_{i \neq j} K_{i j},
$$

so that $L_{0}-H$ becomes a pure constant

$$
L_{0}-H=\left(\lambda_{0}-\alpha_{0}\right) N .
$$

We denote the $\operatorname{sl}(2)$ subalgebra spanned by $\left\{L_{-1}, L_{0}, L_{1}\right\}$ as the "horizontal" $\operatorname{sl}(2)$ algebra to distinguish it from the "vertical" $s l(2)$ algebra of [21] which also acts on the states of the Calogero model and is spanned by the generators

$$
B_{2}^{+}=\frac{1}{2} \sum_{i=1}^{N}\left(a_{i}^{\dagger}\right)^{2}, \quad B_{2}^{0}=H, \quad B_{2}^{-}=\frac{1}{2} \sum_{i=1}^{N}\left(a_{i}\right)^{2} .
$$

Remarkably, the (shifted by a constant) Calogero Hamiltonian serves as the Cartan subalgebra generator of both $s l(2)$ algebras. We have not been able to extend the vertical $\operatorname{sl}(2)$ algebra to a Virasoro algebra. 
The excited states of the Calogero model can be classified both with respect to "vertical" and "horizontal" $s l(2)$ algebras. Our results imply that for the latter case these representations extend to appropriate representations of the whole reductive part of the Virasoro algebra spanned by the $L_{n}$ with $n \leq 1$. It is worth mentioning that both the "vertical" $\operatorname{sl}(2)$ algebra and the "horizontal" Virasoro algebra are generated by symmetric combinations of the Calogero creation and annihilation operators $a_{i}$ and $a_{i}^{\dagger}$ (cf. (23)) so that the Virasoro algebra under consideration does indeed leave invariant the subspace of totally symmetric wavefunctions of the Calogero model.

(2) So far we have used the Calogero realization where the creation and annihilation operators are expressed in terms of the real coordinates $x_{i}$ underlying the Calogero model. However, one can use other representations of the same algebra equally well. For example, the Virasoro commutation relations remain valid for all other representations found in [11]. A particular useful realization is that which for $\nu=0$ reduces to the standard holomorphic representation and thus can be expected to be relevant to conformal field theory. To be specific, consider the "holomorphic realization" of the $S H_{N}(\nu)$ algebra given by:

$$
a_{i}=D_{i}^{z}, \quad a_{i}^{\dagger}=z_{i},
$$

where the $z_{i}$ are $N$ complex coordinates and $D^{z}{ }_{i}$ is the complex Dunkl derivative:

$$
D_{i}^{z}=\frac{\partial}{\partial z_{i}}+\nu \sum_{j \neq i}\left(z_{i}-z_{j}\right)^{-1}\left(1-K_{i j}\right) .
$$

In the holomorphic representation the Virasoro generators take the following form:

$$
L_{\xi}=\sum_{i=1}^{N}\left(\alpha \xi\left(z_{i}\right) D^{z}{ }_{i}+(1-\alpha) D_{i}^{z} \xi\left(z_{i}\right)+\left(\lambda-\frac{1}{2}\right) \frac{\partial}{\partial z_{i}} \xi\left(z_{i}\right)\right) .
$$

Inserting (33) into (34), one can write $L_{\xi}$ as follows:

$$
\begin{aligned}
L_{\xi}= & \sum_{i=1}^{N}\left(\alpha \xi\left(z_{i}\right) \partial_{i}^{K Z}+(1-\alpha) \partial_{i}^{K Z} \xi\left(z_{i}\right)+\left(\lambda-\frac{1}{2}\right) \frac{\partial}{\partial z_{i}} \xi\left(z_{i}\right)\right) \\
& +\nu(1-2 \alpha) \sum_{i \neq j} \xi\left(z_{i}\right) \frac{1}{z_{i}-z_{j}} K_{i j}
\end{aligned}
$$

where the Knizhnik-Zamolodchikov-type derivatives $\partial_{i}^{K Z}$ are defined by

$$
\partial_{i}^{K Z}=\frac{\partial}{\partial z_{i}}+\nu \sum_{j \neq i}\left(z_{i}-z_{j}\right)^{-1}
$$


We observe that for $\alpha=1 / 2$ all $K$-dependent terms vanish. Since the Knizhnik-Zamolodchikov derivatives satisfy the ordinary Heisenberg algebra $\left[\partial_{i}^{K Z}, z_{j}\right]=\delta_{i j}$ one is left, for $\alpha=1 / 2$, with the standard realization of the Virasoro algebra It It is worth mentioning that the fact that the $K$ dependence trivializes for $\alpha=1 / 2$ in the holomorphic representation for the Calogero Hamiltonian was already observed in [7] when discussing the interplay between the Calogero model and anyons.

(3) There exists the following important difference between the horizontal $s l(2)$ algebra (27) and the vertical $s l(2)$ algebra (31) with respect to the dependence of the generators on the center of mass and relative coordinates. In the vertical $s l(2)$ the center of mass degrees of freedom decouple from the relative motion degrees of freedom for arbitrary $N$ in view of the following relation

$$
\sum_{i=1}^{N}\left\{X_{i}, Y_{i}\right\}=\frac{1}{N}\left(\sum_{i<j}^{N}\left\{X_{i}-X_{j}, Y_{i}-Y_{j}\right\}+\left\{\sum_{i=1}^{N} X_{i}, \sum_{j=1}^{N} Y_{j}\right\}\right) .
$$

On the contrary, it turns out that for the horizontal $\operatorname{sl}(2)$ algebra in the holomorphic representation we have a nontrivial mixture of the centre of mass degrees of freedom and the relative motion ones.

The center of mass coordinate $y$ and the relative coordinates $\tilde{z}_{i}$ are defined by

$$
\begin{aligned}
y & =\frac{1}{N} \sum_{i=1}^{N} z_{i}, \quad \tilde{z}_{i}=z_{i}-y \\
\frac{\partial}{\partial y} & =\sum_{i=1}^{N} \frac{\partial}{\partial z_{i}}, \quad \frac{\partial}{\partial \tilde{z}_{i}}=\frac{\partial}{\partial z_{i}}-\frac{1}{N} \frac{\partial}{\partial y}
\end{aligned}
$$

Note that $\sum_{i=1}^{N} \tilde{z}_{i}=\sum_{i=1}^{N} \partial / \partial \tilde{z}_{i}=0$ and that $\partial / \partial \tilde{z}_{i} \tilde{z}_{j}=\delta_{i j}-1 / N$. The expressions for the generators of the horizontal $\operatorname{sl}(2)$ algebra in terms of $y$ and $\tilde{z}_{i}$, when the arbitrary parameters are fixed according to (29), are given

\footnotetext{
${ }^{6}$ One could try to use this observation to give an alternative proof of the existence of the Virasoro algebra with $\alpha=\frac{1}{2}$ for other realizations of the $S H_{N}(\nu)$ algebra. Indeed, if there exists an operator $U$ intertwining the holomorphic and some other representation of the $S H_{N}(\nu)$ algebra (e.g. the Calogero representation we used above) then the proof will become trivial for this other representation as well. Unfortunately, we do not know whether there exists such an operator intertwining between the holomorphic and the Calogero representation. Note that, since $L_{0}$ is the universal Calogero Hamiltonian the knowledge of such an operator $U$ would imply in particular an explicit solution of the Calogero model via reduction to an ordinary harmonic oscillator problem.
} 
by

$$
\begin{aligned}
L_{1} & =\frac{\partial}{\partial y} \\
L_{0} & =y \frac{\partial}{\partial y}+\sum_{i=1}^{N} \tilde{z}_{i} \frac{\partial}{\partial \tilde{z}_{i}}+N\left(\lambda_{0}+\frac{1}{2}-\alpha+\frac{1}{2} \nu(N-1)\right) \\
L_{-1} & =y^{2} \frac{\partial}{\partial y}+2 \sum_{i=1}^{N} y \tilde{z}_{i} \frac{\partial}{\partial \tilde{z}_{i}}+\frac{1}{N} \sum_{i=1}^{N} \tilde{z}_{i}^{2} \frac{\partial}{\partial y}+\sum_{i=1}^{N} \tilde{z}_{i}^{2} \frac{\partial}{\partial \tilde{z}_{i}} \\
& +\nu(1-2 \alpha) \sum_{i \neq j} \tilde{z}_{i} K_{i j}+2 N y\left(\lambda_{0}+\frac{1}{2}-\alpha+\frac{1}{2} \nu(N-1)\right) .
\end{aligned}
$$

We observe that the relative coordinate operators $\tilde{z}_{i}, \partial / \partial \tilde{z}_{i}$ all occur in the $z$ scale-invariant combination $\tilde{z}_{i} \partial / \partial \tilde{z}_{i}$ except for three terms in $L_{-1}$. One of these three terms leads to a nontrivial mixing of the center of mass degrees of freedom and the relative ones in the Calogero model. To get rid of this term, one can consider the limiting procedure where $y \rightarrow y$ and $\tilde{z}_{i} \rightarrow \delta \tilde{z}_{i}$ with $\delta \rightarrow 0$ which does not affect the commutation relations of the $\operatorname{sl}(2)$ algebra as can be checked easily by using (40). We observe that after this limiting procedure the relative coordinate-dependent operators always occur in the combination

$$
\bar{\lambda}=\sum_{i=1}^{N} \tilde{z}_{i} \frac{\partial}{\partial \tilde{z}_{i}}+N\left(\lambda_{0}+\frac{1}{2}-\alpha+\frac{1}{2} \nu(N-1)\right) .
$$

For this particular degenerate realization the relative coordinate operators thus behave as inner coordinates which only effect the conformal weight.

The above limiting procedure can be applied to the whole Virasoro algebra as well. One may verify that after taking the limit $\delta \rightarrow 0$ the generators following from (35) all take the standard form

$$
L_{-n}=y^{n+1} \frac{\partial}{\partial y}+\bar{\lambda}(n+1) y^{n}
$$

with the conformal weight $\bar{\lambda}$ given by (41).

Finally, we note that the center of mass coordinate $y$ plays an important role in defining, in a consistent way, the Virasoro generators $L_{n}(n>0)$ which involve negative powers of $z_{i}$. Specifically, to define inverse powers of $z_{i}$ one first uses the decomposition $z_{i}=y+\tilde{z}_{i}$ and then expands all expressions in powers of the relative coordinates $\tilde{z}_{i}$, e.g. $z_{i}^{-1}=y^{-1} \sum_{n=0}^{\infty}(-)^{n}\left(\frac{\tilde{z}_{i}}{y}\right)^{n}$. In particular, it is convenient to use this approach in deriving the limiting form (42). The same approach is used in the analysis of the $W_{\infty}$-type generalizations which are discussed in section 4. 


\section{Super Extension}

It is natural to extend the results obtained in the previous section to the supersymmetric case, thereby extending the Virasoro algebra to a superVirasoro algebra. Supersymmetric extensions of the Calogero model were recently discussed in [22, 7]. In the following we will frequently make use of the results of [7].

Our starting point is the supersymmetric extension of the $S H_{N}(\nu)$ algebra which is given by the direct product of $S H_{N}(\nu)$ with the Clifford algebra $C_{2 N}$ with generating elements $\theta_{i}$ and $\theta_{i}^{\dagger}$ :

$$
\left\{\theta_{i}, \theta_{j}^{\dagger}\right\}=\delta_{i j} .
$$

The operator $K_{i j}$ is assumed to commute with $\theta_{i}$ and $\theta_{i}^{\dagger}$. Note that the fermionic permutation operators $K_{i j}^{\theta}$ can be realized as [ :

$$
K_{i j}^{\theta}=1-\left(\theta_{i}-\theta_{j}\right)\left(\theta_{i}^{\dagger}-\theta_{j}^{\dagger}\right) .
$$

These operators commute with $a_{i}, a_{i}^{\dagger}$ and have the following standard permutation relations with $\theta_{i}, \theta_{i}^{\dagger}$ :

$$
\theta_{i}^{(\dagger)} K_{i j}^{\theta}=K_{i j}^{\theta} \theta_{j}^{(\dagger)}, \quad\left(K_{i j}^{\theta}\right)^{2}=1 .
$$

In addition, the operators $K_{i j}^{\theta}$ satisfy the properties (2)-(4). One can also define the total permutation operators

$$
K_{i j}^{\mathrm{tot}}=K_{i j} K_{i j}^{\theta}
$$

which exchange both bosonic and fermionic coordinates simultaneously.

In [22, 7] the explicitly supersymmetric form of the super-Calogero Hamiltonian $H_{s}$ was given. In particular, the construction of [7] was based upon an $\operatorname{osp}(1,2)$ supersymmetric extension of the vertical $s l(2)$ algebra

$$
\begin{aligned}
H_{s} & =\left\{Q, Q^{\dagger}\right\} \\
& =\frac{1}{2} \sum_{i=1}^{N}\left\{a_{i}, a_{i}^{\dagger}\right\}+\frac{1}{4} \sum_{i=1}^{N}\left[\theta_{i}, \theta_{i}^{\dagger}\right]+\frac{1}{2} \nu \sum_{i \neq j} K_{i j}^{\mathrm{tot}},
\end{aligned}
$$

where

\footnotetext{
${ }^{7}$ We observe that for $N=2$ the operator $K_{12}^{\theta}$ equals the Klein operator $K$ which occurs in the discussion of the super- $W_{\infty}(\lambda)$ algebra [20]. Indeed, we can write $K=1-2 \theta \theta^{\dagger}$ with $\theta^{(\dagger)} \equiv \frac{1}{\sqrt{2}}\left(\theta_{1}^{(\dagger)}-\theta_{2}^{(\dagger)}\right)$. The Klein operator $K$ satisfies $K^{2}=1$ and anticommutes with $\theta^{(\dagger)}$.

${ }^{8}$ We have reintroduced the frequency parameter $\omega_{F}$ of [7] and taken it to be equal to $\omega_{F}=-\frac{1}{2}$.
} 


$$
Q=\sum_{i=1}^{N} \theta_{i}^{\dagger} a_{i}, \quad Q^{\dagger}=\sum_{i=1}^{N} \theta_{i} a_{i}^{\dagger} .
$$

The generators $Q, Q^{\dagger}$ can be interpreted as odd generators of the $\operatorname{osp}(1,2)$ superextension of the vertical $s l(2)$ algebra. Note that the last term in (47), when acting on the subspace of totally symmetric wavefunctions, reduces to a constant. One may verify that the hamiltonian

$$
H=\frac{1}{2} \sum_{i=1}^{N}\left\{a_{i}, a_{i}^{\dagger}\right\}+\frac{1}{4} \sum_{i=1}^{N}\left[\theta_{i}, \theta_{i}^{\dagger}\right]
$$

satisfies the commutation relations:

$$
\begin{array}{ll}
{\left[H, a_{i}^{\dagger}\right]=a_{i}^{\dagger}, \quad\left[H, a_{i}\right]=-a_{i}} \\
{\left[H, \theta_{i}^{\dagger}\right]=-\frac{1}{2} \theta_{i}^{\dagger},} & {\left[H, \theta_{i}\right]=\frac{1}{2} \theta_{i} .}
\end{array}
$$

In order to describe our Ansatz for the super-Virasoro generators, it is convenient to introduce the "super-Dunkl derivative" $\mathcal{D}_{i}$. In [7] it was shown that there exists a one-parametric class of superderivatives which all fulfill the basic relations

$$
\begin{aligned}
\left\{\mathcal{D}_{i}, \mathcal{D}_{j}\right\} & =2 \delta_{i j} D_{i}^{\theta} \\
\left\{\mathcal{D}_{i}, \theta_{j}\right\} & =\delta_{i j}
\end{aligned}
$$

where $D_{i}^{\theta}$ is some supersymmetric extension of the ordinary (bosonic) Dunkl derivative. All these derivatives were shown in [7] to be related to each other by a similarity transform. Remarkably, it turns out that only one particular member of this class leads to the desired superextension of the Virasoro algebraf?. This particular member is exactly the one which is covariant with respect to the global supersymmetry rules

$$
\delta z_{i}=\theta_{i} \epsilon, \quad \delta \theta_{i}=\epsilon .
$$

To be precise, for the realization of the super-Virasoro algebra, we need the following special derivativeto:

$$
\mathcal{D}_{i}=\mathcal{D}_{i}^{0}+\nu \sum_{j \neq i} \frac{\theta_{i j}}{z_{i j}}\left(1-K_{i j}^{\mathrm{tot}}\right) .
$$

\footnotetext{
${ }^{9}$ Note that the similarity transform of [7] does not leave invariant the Ansatz we use below.

10 This derivative equals the super-Dunkl derivative ${ }^{\alpha} \mathcal{D}_{i}$ of $[7]$ with $\alpha=\nu$.
} 
where

$$
\mathcal{D}_{i}^{0}=\frac{\partial}{\partial \theta_{i}}+\theta_{i} \frac{\partial}{\partial z_{i}}
$$

is the ordinary supercovariant derivative and where we have introduced the supersymmetric line elements

$$
z_{i j}=z_{i}-z_{j}-\theta_{i} \theta_{j}, \quad \theta_{i j}=\theta_{i}-\theta_{j} .
$$

The supercovariant derivatives of these line-elements are given by

$$
\mathcal{D}_{i}^{0} z_{i j}=\mathcal{D}_{j}^{0} z_{i j}=\theta_{i j}, \quad \mathcal{D}_{i}^{0} \theta_{i j}=-\mathcal{D}_{j}^{0} \theta_{i j}=1 .
$$

A noteworthy property of the super-Dunkl derivative $\mathcal{D}_{i}$ is that because of the factor of $\theta_{i}-\theta_{j}$ in front of $K_{i j}^{\text {tot }}$ one can equally well use both $K_{i j}$ and $K_{i j}^{\text {tot }}$ in its definition. The expression for the bosonic derivative $D_{i}^{\theta}$ follows from the anticommutator of two super-Dunkl derivatives and is given by:

$$
D_{i}^{\theta}=\frac{\partial}{\partial z_{i}}+\nu \sum_{l \neq i}\left[\frac{1}{z_{i l}}\left(1-K_{i l}^{\mathrm{tot}}\right)-\frac{\theta_{i l}}{z_{i l}} K_{i l}^{\mathrm{tot}}\left(\mathcal{D}_{i}-\mathcal{D}_{l}-\nu \sum_{j \neq i, j \neq l} \frac{\theta_{l j}}{z_{l j}} K_{l j}^{\mathrm{tot}}\right)\right]
$$

One easily finds that

$$
\left[\mathcal{D}_{i}, z_{j}\right]=\delta_{i j}\left(\theta_{i}+\nu \sum_{k \neq i=1}^{N} \theta_{i k} K_{i k}\right)-\nu \theta_{i j} K_{i j}
$$

This formula is a special case of a more general formula which gives the commutator of $\mathcal{D}_{i}$ with an arbitrary function $f(z, \theta)$ of a single superargument $(z, \theta)$. Using the convention $f_{i} \equiv f\left(z_{i}, \theta_{i}\right)$ and the abbreviation

$$
y_{i j} \equiv \frac{\theta_{i j}}{z_{i j}}
$$

the general formula is given by

$$
\begin{gathered}
{\left[\mathcal{D}_{i}, f_{i}\right]=\mathcal{D}^{0}(f)_{i}+\nu \sum_{i \neq j}\left(f_{i}-f_{j}\right) y_{i j} K_{i j}^{\mathrm{tot}},} \\
{\left[\mathcal{D}_{i}, f_{j}\right]=-\nu\left(f_{i}-f_{j}\right) y_{i j} K_{i j}^{\mathrm{tot}}, \quad i \neq j} \\
\sum_{i}\left[\mathcal{D}_{i}, f_{i}\right]=\sum_{i} \mathcal{D}^{0}(f)_{i} .
\end{gathered}
$$

For the convenience of the reader we give below some useful identities that are obeyed by the quantities $y_{i j}$ : 


$$
\begin{aligned}
y_{i j} & =y_{j i}, \quad y_{i j} y_{k l}=-y_{k l} y_{i j}, \\
y_{i l} y_{l j} & +y_{j l} y_{i j}+y_{i j} y_{i l}=0, \\
\mathcal{D}_{i}^{0} y_{i j} & =-\mathcal{D}_{j}^{0} y_{i j}=\frac{1}{z_{i j}} .
\end{aligned}
$$

We now proceed with our Ansatz for the (super)generators of the $(N=2)$ super Virasoro algebra which is given by

$$
\begin{gathered}
L_{\xi}=\sum_{i}\left(\mathcal{D}_{i} \xi_{i} \mathcal{D}_{i}-\frac{1}{2} \mathcal{D}^{0}(\xi)_{i} \mathcal{D}_{i}+\frac{1}{2} \lambda \xi_{i}^{\prime}\right), \\
Q_{\epsilon}=\sum_{i} \epsilon_{i} \mathcal{D}_{i}+\lambda \sum_{i} \mathcal{D}^{0}(\epsilon)_{i}
\end{gathered}
$$

where $\xi(z, \theta)$ and $\epsilon(z, \theta)$ are arbitrary commuting parameters and $f^{\prime}(z, \theta) \equiv$ $\frac{\partial}{\partial z} f(z, \theta)$.

One can check that the following (anti-)commutation relations are true

$$
\begin{gathered}
\left\{Q_{\epsilon_{1}}, Q_{\epsilon_{2}}\right\}=L_{\xi_{1,2}}, \\
{\left[L_{\xi}, Q_{\epsilon}\right]=Q_{\bar{\epsilon}},}
\end{gathered}
$$

where

$$
\xi_{1,2}=2 \epsilon_{1} \epsilon_{2}
$$

and

$$
\bar{\epsilon}=\xi \epsilon^{\prime}-\frac{1}{2} \epsilon \xi^{\prime}+\frac{1}{2} \mathcal{D}^{0}(\xi) \mathcal{D}^{0}(\epsilon) .
$$

The proof of the anti-commutation relation (68) is relatively simple. To illustrate how it works we consider the case with $\lambda=0$. A straightforward calculation gives

$$
Q_{\epsilon} Q_{\epsilon}=\sum_{i, j} \epsilon_{i} \mathcal{D}_{i} \epsilon_{j} \mathcal{D}_{j}=\sum_{i, j}\left(\epsilon_{i} \epsilon_{j} \mathcal{D}_{i} \mathcal{D}_{j}+\epsilon_{i}\left[\mathcal{D}_{i}, \epsilon_{j}\right] \mathcal{D}_{j}\right)
$$

Taking into account the fact that the parameter $\epsilon$ is commuting, the basic anti-commutation relation (52) of the super-Dunkl derivatives and the formulae (62), (63) one obtains after some simple algebra

$$
Q_{\epsilon} Q_{\epsilon}=\sum_{i}\left(\epsilon_{i}^{2} \mathcal{D}_{i}^{2}+\epsilon_{i} \mathcal{D}_{i}^{0}\left(\epsilon_{i}\right) \mathcal{D}_{i}\right)+\nu \sum_{i \neq j} \epsilon_{i}^{2} y_{i j} K_{i j}^{\mathrm{tot}}\left(\mathcal{D}_{i}-\mathcal{D}_{j}\right)
$$

Analogously one finds

$$
\sum_{i} \mathcal{D}_{i} \epsilon_{i}^{2} \mathcal{D}_{i}=\sum_{i}\left(\epsilon_{i}^{2} \mathcal{D}_{i}^{2}+\mathcal{D}_{i}^{0}\left(\epsilon_{i}^{2}\right) \mathcal{D}_{i}\right)+\nu \sum_{i \neq j} \epsilon_{i}^{2} y_{i j} K_{i j}^{\text {tot }}\left(\mathcal{D}_{i}-\mathcal{D}_{j}\right)
$$


Combining (73) with (74) proves the relation (68) for $\lambda=0$. It is simple to generalize this relation to non-vanishing values of $\lambda$.

The proof of the $\left[L_{\xi}, Q_{\epsilon}\right]$ commutator is much more involved and is described in appendix B. Let us note that as in the bosonic case the fact that the generators $Q$ and $L$ form a closed super Virasoro algebra is representation independent, i.e. it follows only from the basic commutation relations $(52),(53)$ and (60). Note that from the relations (68) and (69) it follows that the $L$ generators satisfy the commutation relations of the Virasoro algebra.

Like in the bosonic case $L_{0}$ can be identified with a $Z$-grading operator by adjusting the $\lambda$ parameter as follows:

$$
\lambda=\lambda_{0}+\nu N^{-1} \sum_{i \neq j}\left(K_{i j}^{\mathrm{tot}}-1\right) .
$$

The generators $L_{1}, L_{0}$ and $L_{-1}$ of the $s l_{2}$ subalgebra of Virasoro which correspond respectively to the parameters $\xi=1, z$, and $z^{2}$ in (66) then take the form

$$
\begin{gathered}
L_{1}=\sum_{i=1}^{N} \frac{\partial}{\partial z_{i}} \\
L_{0}=\sum_{i} z_{i} \frac{\partial}{\partial z_{i}}+\frac{1}{2} \sum_{i} \theta_{i} \frac{\partial}{\partial \theta_{i}}+\frac{1}{2} \lambda_{0} N \\
L_{-1}=\sum_{i} z_{i}^{2} \frac{\partial}{\partial z_{i}}+\sum_{i} z_{i} \theta_{i} \frac{\partial}{\partial \theta_{i}}+\lambda_{0} \sum_{i=1}^{N} z_{i}-\nu \sum_{i \neq j} z_{i} K_{i j}^{\mathrm{tot}} \\
+\nu N^{-1} \sum_{i \neq j} K_{i j}^{\mathrm{tot}} \sum_{l=1}^{N} z_{l}+\nu^{2} \sum_{j \neq k \neq i} z_{i}^{2} y_{i j} K_{i j}^{\mathrm{tot}} y_{j k} K_{j k}^{\mathrm{tot}}
\end{gathered}
$$

The $\nu^{2}$ contribution to $L_{0}$ from the last term in the representation (91) for the Virasoro generators vanishes due to the following identity

$$
z_{l} y_{i l} y_{l j}+z_{j} y_{j l} y_{i j}+z_{i} y_{i j} y_{i l}=0
$$

The fermionic coordinates in $L_{0}$ only appear as a shift of the conformal parameter $\lambda_{0}$ which involves the fermionic number operator $\sum_{i} \theta_{i} \frac{\partial}{\partial \theta_{i}}$. This is not the case for the $L_{1}$ generator which involves non-trivial mixings between the relative and fermionic coordinates.

An interesting distinction between the bosonic and fermionic case is that the supergenerators involve only one independent free parameter, the conformal weight $\lambda$. Due to the identity (64) a term of the form $\sum_{i} \mathcal{D}_{i} \epsilon_{i}$ can be rewritten in terms of $\sum_{i} \epsilon_{i} \mathcal{D}_{i}$ modulo a shift in the parameter $\lambda$. Therefore, in the supersymmetric case, there is no room for a second free parameter, like the parameter $\alpha$ in the bosonic case. 
When restricted to the subspace of $\theta$-independent functions, the above generators of the $s l_{2}$ subalgebra of the super-Virasoro algebra coincide with the bosonic generators (27) for $\alpha=1$ ( and with the conformal weight parameters identified as $\lambda$ (bosonic) $-1 / 2=1 / 2 \lambda$ (fermionic) ) except for the $\nu^{2}$-dependent term in $L_{-1}$. This term contains an explicit $\theta$-dependence. Therefore, for $\nu \neq 0$, one cannot truncate the $s l_{2}$ subalgebra of the superVirasoro algebra to the sector of operators acting on $\theta$-independent functions.

The super-Virasoro algebra under consideration is formulated here in terms of $N=1$ superfield parameters analogous to the one-particle formulation given in [20]. The algebra however is $N=2$ supersymmetric as can easily be seen from the component analysis: the superfield generators $L_{\xi}$ and $Q_{\epsilon}$ involve one spin 2 current, two spin $3 / 2$ currents (one from $L_{\xi}$, and another one from $Q_{\epsilon}$ ), and one spin 1 current (the $\theta$ - component in $Q_{\epsilon}$ ). In particular, the $u(1)$ component of the spin 1 current has the form

$$
J_{0}=\sum_{i} \theta_{i} \frac{\partial}{\partial \theta_{i}}-\nu \sum_{i \neq j} \frac{\theta_{i} \theta_{j}}{z_{i j}}\left(1-K_{i j}^{\mathrm{tot}}\right) .
$$

Along with the corresponding supergenerators the above spin 2 and spin 1 generators form the "horizontal" $\operatorname{osp}(2 \mid 2)$ subalgebra of the full super Virasoro algebra. This algebra should be distinguished from the "vertical" $\operatorname{osp}(2 \mid 2)$ algebra which was considered in [7].

\section{Conclusions}

In this paper we have shown that based on the Calogero model inspired algebras $S H_{N}(\nu)$ and their superextensions one can construct new realizations of the (super-) Virasoro algebra analogous to the standard realization by (super) vector fields based on the ordinary Heisenberg algebras (or, equivalently, the algebra of differential operators). The algebra $S H_{N}(\nu)$ can be regarded as the associative algebra with generating elements $a_{i}^{\dagger}, a_{j}$ and $K_{i j}$. One can consider the same algebra but now with the (super-) commutator as the product law. This leads to infinite-dimensional Lie (super)algebras which we denote by $W_{N, \infty}(\nu)$ in analogy with the construction of ordinary $W_{\infty}$-type algebras via commutators of elements of the enveloping algebra of the Heisenberg algebra [12, 23, 24]. Due to the results of this paper these algebras contain the Virasoro subalgebra and therefore can be regarded as an extended conformal algebra thereby justifying the name $W_{\infty}$-type algebra. Note that the $W_{1+\infty}$ algebra can be identified with the algebra $W_{1, \infty}(\nu)$ (the parameter $\nu$ drops out for $N=1$ ).

The $W_{N, \infty}(\nu)(N \geq 2)$ algebras are rather large algebras involving an infinite number of higher spin generators of each spin. An interesting question is whether there exists truncations of the $W_{N, \infty}(\nu)$ algebra that still contain 
the Virasoro algebra. There indeed exists such a truncated algebra. This subalgebra is spanned by the symmetric elements $w$ that commute with the permutation operators, i.e.

$$
\left[K_{i j}, w\right]=0 .
$$

We denote this subalgebra of $W_{N, \infty}(\nu)$ by $\tilde{W}_{N, \infty}(\nu)$. Since the Virasoro generators themselves obey the symmetry conditions $(23), \tilde{W}_{N, \infty}(\nu)$ contains the Virasoro algebra as a subalgebra. The algebra $\tilde{W}_{N, \infty}(\nu)$ contains infinitely many higher-spin currents of each spin.

A natural question to ask is whether $W_{1+\infty}$ is a proper subalgebra of $W_{N, \infty}(\nu)$. The answer to this question seems to be negative. So far our attempts to embed $W_{1+\infty}$ into $W_{N, \infty}(\nu)$ as a proper subalgebra were not successful.

The above results can be naturally extended to the supersymmetric case. One can define the superalgebras $S W_{N, \infty}(\nu)$ and $S \tilde{W}_{N, \infty}(\nu)$ based on the superextensions considered in section 3. The superalgebra $S \tilde{W}_{N, \infty}(\nu)$ is spanned by the symmetric elements obeying the conditions

$$
\left[K_{i j}^{\mathrm{tot}}, w\right]=0 .
$$

We believe that the generalized $W_{\infty}$-type algebras introduced above may have interesting applications in the context of conformal field theory, integrable systems and the quantum Hall effect. We hope to discuss some of these applications in a future publication.

\section{Acknowledgments}

We thank L. Brink, S. Isakov, J. Leinaas, and A. Polychronakos for useful discussions. The work of E.B. has been made possible by a fellowship of the Royal Netherlands Academy of Arts and Sciences (KNAW). One of us (M.V.) would like to thank Groningen University for hospitality.

\section{Appendix A: Proof of the identities (14), (15) and (16)}

To prove the identities (14), (15) and (16) we need a particular case of (17) when $f\left(a^{\dagger}\right)$ is a function of one argument $a_{i}^{\dagger}$ for some fixed $i$, i.e. $f\left(a^{\dagger}\right)=$ $\phi\left(a_{i}^{\dagger}\right)$. For this particular case one easily derives from (17)

$$
\left[a_{i}, \phi\left(a_{j}^{\dagger}\right)\right]=\delta_{i j} \phi^{\prime}\left(a_{i}^{\dagger}\right)+\nu \delta_{i j} \sum_{l=1}^{N}\left(a_{i}^{\dagger}-a_{l}^{\dagger}\right)^{-1}\left(\phi\left(a_{i}^{\dagger}\right)-\phi\left(a_{l}^{\dagger}\right)\right) K_{i l}
$$




$$
-\nu\left(a_{i}^{\dagger}-a_{j}^{\dagger}\right)^{-1}\left(\phi\left(a_{i}^{\dagger}\right)-\phi\left(a_{j}^{\dagger}\right)\right) K_{i j},
$$

where $\phi^{\prime}(x)=\frac{\partial}{\partial x} \phi(x)$. Note that the commutation relations (1) are themselves a particular case of (83) for $\phi(x)=x$.

The proof of (14) and (15) is straightforward by observing that the two $\nu$-dependent terms coming from the right-hand-side of (83) cancel against each other after the substitution of (83) into (14), (15). The $\nu$-independent part of the resulting expression gives the right-hand-side of (14), (15).

To prove the identity (16) it is convenient to decompose the left-hand-side of $(16)$,

$$
X \equiv \sum_{i, j=1}^{N}\left[\left[a_{i}, \xi_{1}\left(a_{i}^{\dagger}\right)\right],\left[a_{j}, \xi_{2}\left(a_{j}^{\dagger}\right)\right]\right]
$$

into three parts $X^{(0)}, X^{(1)}$ and $X^{(2)}$, which are, respectively, independent of $\nu$, linear in $\nu$ and quadratic in $\nu$. Due to (83) we obviously have $X^{(0)}=0$. Using the shorthand notation $\xi_{1 i}=\xi_{1}\left(a_{i}^{\dagger}\right)$ and $\xi_{2 i}=\xi_{2}\left(a_{i}^{\dagger}\right)$ we can write

$$
\begin{aligned}
X^{(1)} & =\nu \sum_{i=1}^{N}\left[\xi_{1 i}^{\prime}, \sum_{l \neq i}\left(a_{i}^{\dagger}-a_{l}^{\dagger}\right)^{-1}\left(\xi_{2 i}-\xi_{2 l}\right) K_{i l}\right]-(1 \leftrightarrow 2) \\
& =\nu \sum_{l \neq i}\left(\xi_{1 i} \prime-\xi_{1 l}^{\prime}\right)\left(a_{i}^{\dagger}-a_{l}^{\dagger}\right)^{-1}\left(\xi_{2 i}-\xi_{2 l}\right) K_{i l}-(1 \leftrightarrow 2),
\end{aligned}
$$

which also vanishes because the summand is antisymmetric under the interchange $i \leftrightarrow l$.

Finally, for $X^{(2)}$ one gets from 83 )

$$
X^{(2)}=\nu^{2} \sum_{i, l, n}\left[\left(a_{i}^{\dagger}-a_{l}^{\dagger}\right)^{-1}\left(\xi_{1 i}-\xi_{1 l}\right) K_{i l},\left(a_{i}^{\dagger}-a_{n}^{\dagger}\right)^{-1}\left(\xi_{2 i}-\xi_{2 n}\right) K_{i n}\right] .
$$

The contribution to the summation of the terms with $l=n$ vanish so that one is left with

$$
\begin{aligned}
X^{(2)} & =\nu^{2} \sum_{i \neq l, i \neq n, l \neq n}\left(\left(a_{i}^{\dagger}-a_{l}^{\dagger}\right)^{-1}\left(\xi_{1 i}-\xi_{1 l}\right)\left(a_{l}^{\dagger}-a_{n}^{\dagger}\right)^{-1}\left(\xi_{2 l}-\xi_{2 n}\right) K_{i l} K_{i n}\right. \\
& \left.-\left(a_{i}^{\dagger}-a_{n}^{\dagger}\right)^{-1}\left(\xi_{2 i}-\xi_{2 n}\right)\left(a_{n}^{\dagger}-a_{l}^{\dagger}\right)^{-1}\left(\xi_{1 n}-\xi_{1 l}\right) K_{i n} K_{i l}\right) .
\end{aligned}
$$

After replacing $l \leftrightarrow n$ in the second term we can write $X^{(2)}$ as

$$
\begin{aligned}
X^{(2)}= & \nu^{2} \sum_{\substack{i \neq l, i \neq n, l \neq n\\
}}\left(a_{i}^{\dagger}-a_{l}^{\dagger}\right)^{-1}\left(a_{l}^{\dagger}-a_{n}^{\dagger}\right)^{-1}\left(a_{i}^{\dagger}-a_{n}^{\dagger}\right)^{-1} \\
& \left\{\left(a_{i}^{\dagger}-a_{n}^{\dagger}\right)\left(\xi_{1 i} \xi_{2 l}+\xi_{1 l} \xi_{2 n}-\xi_{1 i} \xi_{2 n}\right)-1 \leftrightarrow 2\right\} K_{i l} K_{i n} .
\end{aligned}
$$


Finally, using the cyclic property (2) of the elementary permutation generators one easily verifies by permuting the summation indices $i, j, l$ that the $\left(a_{i}^{\dagger}-a_{n}^{\dagger}\right)$ coefficient in the second line can be replaced by $1 / 3\left[\left(a_{i}^{\dagger}-a_{n}^{\dagger}\right)+\right.$ $\left.\left(a_{l}^{\dagger}-a_{i}^{\dagger}\right)+\left(a_{n}^{\dagger}-a_{l}^{\dagger}\right)\right]$ which is identically zero. This concludes the proof of the identity (16).

In addition to the identities proven above there also exist the following useful identities which can be proven analogously

$$
\begin{gathered}
\sum_{i=1}^{N}\left\{\left[a_{i},\left(\xi_{1}\left(a_{j}^{\dagger}\right)\right], \xi_{2}\left(a_{i}^{\dagger}\right)\right\}=2 \xi_{2}\left(a_{j}^{\dagger}\right) \xi_{1}^{\prime}\left(a_{j}^{\dagger}\right),\right. \\
\sum_{i, j=1}^{N}\left[\left[a_{i}, \xi_{1}\left(a_{j}^{\dagger}\right)\right],\left[a_{j}, \xi_{2}\left(a_{i}^{\dagger}\right)\right]\right]=0 .
\end{gathered}
$$

\section{Appendix B: The sketch of calculation of $\left[L_{\xi}, Q_{\epsilon}\right]$}

Below we outline the main steps of our calculation of the $\left[L_{\xi}, Q_{\epsilon}\right]$ commutator for the case with $\lambda=0$.

It is convenient to work with the following form for the Ansatz of the $L_{\xi^{-}}$ generator which can easily be obtained from (66) with the aid of the formula (59):

$$
L_{\xi}=\sum_{i} \xi_{i} D_{i}^{\theta, 0}+\frac{1}{2}\left(\mathcal{D}_{i}^{0} \xi_{i}\right) \mathcal{D}_{i}+\nu^{2} \sum_{j \neq k \neq i} \xi_{i} y_{i j} K_{i j}^{\mathrm{tot}} y_{j k} K_{j k}^{\mathrm{tot}}
$$

with

$$
D_{i}^{\theta, 0} \equiv \frac{\partial}{\partial z_{i}}+\nu \sum_{j \neq i} \frac{1}{z_{i j}}\left(1-K_{i j}^{\mathrm{tot}}\right) .
$$

As a first stage we find for the commutator:

$$
\begin{aligned}
& {\left[L_{\xi}, Q_{\epsilon}\right]=\sum_{i, j}\left(\xi_{i}\left[D_{i}^{\theta, 0}, \epsilon_{j}\right] \mathcal{D}_{j}+\xi_{i} \epsilon_{j}\left[D_{i}^{\theta, 0}, \mathcal{D}_{j}\right]-\epsilon_{i}\left[\mathcal{D}_{i}, \xi_{j}\right] D_{j}^{\theta, 0}\right.} \\
& \left.+\frac{1}{2}\left(\mathcal{D}_{i}^{0} \xi_{i}\right)\left[\mathcal{D}_{i}, \epsilon_{j}\right] \mathcal{D}_{j}+\frac{1}{2} \epsilon_{j}\left(\mathcal{D}_{i}^{0} \xi_{i}\right)\left\{\mathcal{D}_{i}, \mathcal{D}_{j}\right\}-\frac{1}{2} \epsilon_{j}\left\{\mathcal{D}_{j},\left(\mathcal{D}_{i}^{0} \xi_{i}\right)\right\} \mathcal{D}_{i}\right) \\
& +\nu^{2} \sum_{i \neq j \neq k} \sum_{l}\left[\xi_{i} y_{i j} K_{i j}^{\mathrm{tot}} y_{j k} K_{j k}^{\mathrm{tot}}, \epsilon_{l} \mathcal{D}_{l}\right] .
\end{aligned}
$$

We first consider the terms in the lowest order in $\nu$ which are of the form $\xi \epsilon^{\prime} \mathcal{D}_{i}, \epsilon \xi^{\prime} \mathcal{D}_{i},\left(\mathcal{D}_{i}^{0} \xi_{i}\right)\left(\mathcal{D}_{i}^{0} \epsilon_{i}\right) \mathcal{D}_{i}$ and $\epsilon_{i}\left(\mathcal{D}_{i}^{0} \xi_{i}\right) D_{i}^{\theta, 0}$. The first three type of terms gives us the required supersymmetry transformation with the parameter (71). The last type of term cancels $\square$. We next consider the remaining terms linear

${ }^{11}$ In doing the calculation there is no need to write out the derivatives $\mathcal{D}_{i}$ and $D_{i}^{\theta, 0}$ in terms of a $\nu$-independent part and terms linear in $\nu$. Therefore, strictly speaking, our calculation also takes care of a class of $\nu^{2}$-dependent terms in the commutator. 
in $\nu$. They only come from the first and second line in (93). We observe that all $\nu$-dependent terms in the second line of (93) are proportional to $\left(\mathcal{D}_{i}^{0} \xi_{i}\right)$, whereas all terms in the first line are proportional to $\xi_{i}$. This implies that the remaining $\nu$-dependent terms occurring in the first and second line should cancel independently. The second line in (93) gives rise to terms of the form ${ }^{\mathbb{2}}$

$$
\nu \frac{\theta_{i j}}{z_{i j}}\left(\mathcal{D}_{i}^{0} \xi_{i}\right) \epsilon_{i} K_{i j}^{\mathrm{tot}} \mathcal{D}_{i}
$$

These terms can be shown to cancel amongst each other without the need to write out the extra $\nu^{2}$-dependent terms occurring inside $\mathcal{D}_{i}$ explicitly. Therefore, this cancellation also involves a class of terms quadratic in $\nu$.

We next consider the terms linear in $\nu$ coming from the first line in (93). The following three types of terms linear in $\nu$ occur:

$$
\nu \frac{\theta_{i j}}{\left(z_{i j}\right)^{2}} \xi_{i} \epsilon_{j}\left(1-K_{i j}^{\mathrm{tot}}\right), \quad \nu \frac{\theta_{i j}}{z_{i j}} \xi_{i} \epsilon_{j} K_{i j}^{\mathrm{tot}} \frac{\partial}{\partial z_{i}}, \quad \nu \frac{1}{z_{i j}} \xi_{i} \epsilon_{j} K_{i j}^{\mathrm{tot}} \mathcal{D}_{i}^{0} .
$$

All three type of terms can be shown to cancel amongst each other.

We now consider the terms quadratic in $\nu$. In the second line of (93) only the anticommutator in the second term leads to a $\nu^{2}$-dependent term which has not yet been considered. This term cancels against a $\operatorname{similar}\left(\mathcal{D}_{i}^{0} \xi_{i}\right)$ dependent term coming from the commutator in the third line of (93). This finishes the discussion of the second line in (93): all terms have either been cancelled or contribute to the supersymmetry parameter $\bar{\epsilon}$ given in (71). All remaining $\nu^{2}$-dependent terms come from the first and second line in (93). The following two type of terms occur:

$$
\nu^{2} \frac{\theta_{i j}}{\left(z_{i j}\right)^{2}} \xi_{i} \epsilon_{j} K_{i j}^{\mathrm{tot}}, \quad \nu^{2} \frac{\theta_{i j}}{\left(z_{i j}\right)^{2}} \xi_{i} \epsilon_{j} K_{i j}^{\mathrm{tot}} K_{j k}^{\mathrm{tot}} .
$$

The terms linear in $K_{i j}^{\text {tot }}$ only come from the first line in (93) and it can be shown that they cancel amongst each other. We next consider the terms quadratic in $K_{i j}^{\text {tot }}$. Once we have moved the permutation operators to the right we may distinguish between two type of terms which should cancel independently of each other. The first type of terms are proportional to $\xi_{i} \epsilon_{i}$, i.e. $\xi$ and $\epsilon$ have the same index; the second type of terms are proportional to $\xi_{i} \epsilon_{j}$ with $i \neq j$. Both type of terms can be further subdivided into either terms with a summation over three different indices $i \neq j \neq k$ or terms with a summation over two different indices $i \neq j$. A straightforward but somewhat tedious calculation shows that all type of terms cancel amongst each other.

We finally consider the terms trilinear in $\nu$. They only come from the commutator in the third line of (93) and are given by

\footnotetext{
${ }^{12}$ We only give the general structure of the terms. We use a notation where the specific name of the indices is irrelevant.
} 


$$
\nu^{3} \sum_{i \neq j \neq k} \sum_{l \neq m}\left[\epsilon_{l}\left(y_{l m} K_{l m}^{\mathrm{tot}}\right), \xi_{i}\left(y_{i j} K_{i j}^{\mathrm{tot}}\right)\left(y_{j k} K_{j k}^{\mathrm{tot}}\right)\right] .
$$

We first consider the case that either $l$ or $m$ is equal to $i, j$ or $k$ but not both. The terms in the commutator corresponding to this case can always be written into the following three type of terms 5 :

$$
\nu^{3} \sum_{i \neq j \neq k \neq l}\left(\epsilon_{i} \xi_{i} y_{i j} y_{i k} y_{i l} \times K^{3}, \quad \epsilon_{i} \xi_{j} y_{i j} y_{j k} y_{j l} \times K^{3}, \quad \epsilon_{i} \xi_{j} y_{i j} y_{j k} y_{i l} \times K^{3}\right) .
$$

To do this one must make use of the $y$-identities given in (65) A tedious calculation shows that all three type of terms cancel. Finally, we consider the case that both $l$ and $m$ are equal to $i, j$ or $k$. It can be easily shown that this case always leads to terms which identically vanish.

It is straightforward to extend the calculation of the $\left[L_{\xi}, Q_{\epsilon}\right]$ commutator to non-zero values of $\lambda$.

\section{References}

[1] F. Calogero, J. Math. Phys. 10 (1969) 2191, 2197.

[2] F. Calogero, J. Math. Phys. 12 (1971) 419.

[3] A.P. Polychronakos, Phys. Rev. Lett. 69 (1992) 703.

[4] L. Brink, T.H. Hansson and M.A. Vasiliev, Phys. Lett. B286 (1992) 109.

[5] T. H. Hansson, J. M. Leinaas, and J. Myrheim, Nucl. Phys. B384 (1992) 559

[6] A.P. Polychronakos, Phys. Lett. B264 (1991) 362.

[7] L. Brink, T. H. Hansson, S. E. Konstein and M. A. Vasiliev, Nucl. Phys. B401 (1993) 591.

[8] J. M. Leinaas, and J. Myrheim, Nuovo Cimento, B37, 1977, p. 1 - 37.

[9] F. Wilczek, Phys. Rev. Lett. 48, (1982), 1144.

[10] R. B. Laughlin, The Quantum Hall Effect, edited by R. E. Prange, E. Girvin, and M. Steven (Springer-Verlag, New York, 1990); and references therein.

\footnotetext{
${ }^{13}$ The specific name of the indices in this formula is important now.
} 
[11] L. Brink and M.A. Vasiliev, Mod. Phys. Lett. A8 (1993) 3585.

[12] M. A. Vasiliev, Fortschr. Phys. 36 (1988) 33.

[13] C.N. Pope, L.J. Romans and X. Shen, Phys. Lett. B242 (1990) 401.

[14] A. Cappelli, C. A. Trugenberger, and G. R. Zemba Nucl. Phys. B396 (1993) 465; Phys. Lett. B306 (1993) 100; Phys. Rev. Lett. 72 (1994) 1902; and references therein.

[15] V. Kac and A. Radul, Commun. Math. Phys. 157 (1993) 429.

[16] M.A. Olshanetsky and A.M. Perelomov, Phys. Rep. 71 (1981) 313; ibid. Phys. Rep. 94 (1983) 313.

[17] C.F. Dunkl, Trans. Am. Math. Soc. 311 (1989) 167.

[18] J.A. Minahan and A.P. Polychronakos, Phys. Lett. B302 (1993) 265.

[19] O. Dodlov, S. Konstein, and M.A. Vasiliev, JETPh Lett. 58 (1993) 911.

[20] E. Bergshoeff, B. de Wit and M.A. Vasiliev, Nucl. Phys. B366 (1991) 315.

[21] A.M. Perelomov, Teor. Mat. Fiz. 6 (1971) 364.

[22] D.Z. Freedman and P. Mende, Nucl. Phys. B344 (1990) 317.

[23] E. Bergshoeff, M.P. Blencowe and K.S. Stelle, Commun. Math. Phys. 128 (1990) 213.

[24] C.N. Pope, L.J. Romans and X. Shen, Nucl. Phys. B339 (1990) 191. 